\title{
Performance Analysis of Multicast Video Streaming in IEEE 802.11 b/g/n Testbed Environment
}

\author{
Aleksander Kostuch ${ }^{1}$, Krzysztof Gierłowski ${ }^{2}$, and Jozef Wozniak ${ }^{2}$ \\ ${ }^{1}$ Sprint Sp. z o.o., Gdansk, Poland \\ ${ }^{2}$ Gdansk University of Technology, Poland \\ Aleksander.Kostuch@sprint.pl, \{gierk, jowoz\}@eti.pg.gda.pl
}

\begin{abstract}
The aim of the work is to analyse capabilities and limitations of different IEEE 802.11 technologies (IEEE $802.11 \mathrm{~b} / \mathrm{g} / \mathrm{n}$ ), utilized for both multicast and unicast video streaming transmissions directed to mobile devices. Our preliminary research showed that results obtained with currently popular simulation tools can be drastically different than these possible in real-world environment, so, in order to correctly evaluate performance of video streaming, a simple wireless test-bed infrastructure has been created. The results show a strong dependence of the quality of video streaming on the chosen transmission technology. At the same time there are significant differences in perception quality between multicast (1:n) and unicast (1:1) streams, and also between devices offered by different manufacturers. The overall results seem to demonstrate, that, while multicast support quality in different products is still varied and often requires additional configuration, it is possible to select a WiFi access point model and determine the best system parameters to ensure a good video transfer conditions in terms of acceptable QoP/E (Quality of Perception/Exellence).
\end{abstract}

Keywords: Performance, analysis, video, IEEE $802.11 \mathrm{~b} / \mathrm{g} / \mathrm{n}$, multicast, unicast, wifi, wireless, mos, qop, dcf, access point.

\section{Introduction}

Until recently, computer networks were mostly used for bulk data transfers (for example: digital files), generated by various applications. However, recently we have been witnessing a dramatic growth of interest in real-time multimedia transfers, especially with audio and video content. Successful transmission of such content over computer packet networks require specialised client-side mechanisms, in order to adjust transmission parameters and ensure appropriate transport and application protocol configuration to obtain required reception quality. Also, requirements regarding Quality of Service (QoS) in the network itself, necessary to support real-time streaming traffic, are much more restrictive and difficult to provide.

Obtaining such QoS support in case of classical, cable-based network technologies is a fairly well researched and documented task, comparatively easy due to high available bandwidth and stable nature of transmission medium.

Growing popularity of WiFi networks creates a natural need to provide the same services in wireless environment. Here the same task is much more difficult. The changing 
and unpredictable transmission medium creates a very difficult environment for QoSrealted network mechanisms. Also, differences between particular implementations of network hardware tend to be much more prominent than in case of cable-based solutions, and can result in drastically different performance in similar conditions.

At the same time, the required high quality of video transmissions is dictated by the high expectations of end-users (clients), thus quality of network service available for audio/video streaming transmissions, delivered by any wireless network connectivity provider, becomes one of the crucial issues.

Recently some interesting studies were published concerning video and multicast over 802.11 networks. Research presented in [2] and [4] discussed the topic of maximizing number of users, balancing the load among APs and minimizing the load of APs. Authors based their research on multicast traffic in WLAN environment on simulations only. Different research presented in [8] indicated the influence of network streaming quality on MOS (Mean Opinion Score) in case of $25 \mathrm{fps}$ (frame per second) video content. MOS was about 4,5 when movie was transmitted with a very small jitter. It dropped to about 3,5 when the transmission experienced a single long freeze/skip. MOS was still lower $(2,5)$ when frequent short freezes/skips occurred. Moreover, the value of MOS has been proved to decrease when decrease frame rate - MOS was about 4 when movie streamed at 12,5 fps and 2,3 at $5 \mathrm{fps}$. In their research authors used H.264 codec (stream bandwidth about $1 \mathrm{Mbps}$ ) and WMV player version 9.

On the basis of these publications, we decided to concentrate our experiments on the popular (MPEG-4/H.263) codec and its well known usage scenarios, which are widely accepted in commercial networks.

Experiments similar to ours, but only in IEEE 802.11 b environment are described in [9]. The measurements of network bandwidth show that such network is too slow for the transmission of high definition video streams which proved to be fully consistent with our own results. Authors in [5] focused their experiments on multicast streaming in $802.11 \mathrm{~g}$ environment, but have not employed any subjective quality assessment methods.

In our paper we investigate different IEEE 802.11 technologies (IEEE 802.11 $\mathrm{b} / \mathrm{g} / \mathrm{n}$ ), looking for the most beneficial operational parameters and configurations of WLAN networks for video streaming transfers. The variety of possible WLAN configurations and WiFi standards creates a need to determine their capabilities and to estimate the video stream transmission quality.

The paper is organized in the following way. In the following section (Digital Video Transmission) we present an overview of basic mechanisms of IP-based video transmissions, together with a short overview of MPEG standards. Differences in unicast (1:1) and multicast $(1: n)$ streaming, are also briefly discussed. In the next section our test-bed environment and methods of quality analysis (in our case Quality of Perception / Excelence - QoP/E) are described, followed by Results section, containing discussion of obtained results, together with some comments and recommendations. The article is concluded with their summarization and plans of future research.

\section{Digital Video Transmission}

Parameters (such as resolution, number of frames displayed per second, color depth etc.) specified in current TV standards (see Table 1), starting from aged SECAM and 
NTSC and ending with Full HD digital TV, make it very ineffective to even try to transmit over the network the video signal in its base, unmodified form. Bandwidth requirements would clearly be unacceptable and precise timing relations would be impossible to meet without allocation of a very large buffer space.

Table 1. Popular television standards

\begin{tabular}{|c|c|c|c|c|}
\hline \multirow{2}{*}{ System } & \multicolumn{2}{|c|}{ Resolution } & \multicolumn{2}{c|}{ Frequency of image fields changes } \\
\cline { 2 - 5 } & $\begin{array}{c}\text { Number of } \\
\text { lines }\end{array}$ & $\begin{array}{c}\text { Numbers of } \\
\text { points in line }\end{array}$ & $\begin{array}{c}\text { Refresh rate } \\
{[\mathrm{Hz}]}\end{array}$ & $\begin{array}{c}\text { Number of video } \\
\text { frames per second }\end{array}$ \\
\hline $\begin{array}{c}\text { SECAM } \\
\text { fr. Colour } \\
\text { electronic system } \\
\text { with memory) }\end{array}$ & 625 & 720 & 50 & 25 \\
\hline $\begin{array}{c}\text { NTSC } \\
\text { (National } \\
\text { Television } \\
\text { System } \\
\text { Committee) }\end{array}$ & 525 & $\begin{array}{c}720 \text { or } 320 \\
\text { (VHS) }\end{array}$ & 59,94 & 29,97 \\
\hline $\begin{array}{c}\text { PAL } \\
\text { (Phase } \\
\text { Alternating Line) }\end{array}$ & 625 & $\begin{array}{c}720 \text { or 320 } \\
\text { (VHS) }\end{array}$ & 50 & 25 \\
\hline $\begin{array}{c}\text { CCTV (Closed- } \\
\text { Circuit } \\
\text { TeleVision) }\end{array}$ & 288 & 360 & 60 & 30 \\
\hline $\begin{array}{c}\text { Ready for } \\
\text { HDTV }\end{array}$ & 1280 & 720 & 50 & 25 \\
\hline Full HD & 1920 & 1080 & 50 & 25 \\
\hline
\end{tabular}

It is evident, that video information needs to be encoded in order to minimize the amount of traffic, to effectively transport it across a network system. Because of that requirement, development of efficient encoding techniques has an immense influence on the popularity of computers employed as audio-video systems [11]. Encoding mechanisms often include compression or reduction of primary video information [6].

Table 2. Characteristics of popular encoding standards

\begin{tabular}{|l|c|c|l|}
\hline Codec name & $\begin{array}{l}\text { Bandwidth } \\
\text { necessary for PAL }\end{array}$ & $\begin{array}{l}\text { Error } \\
\text { sensitivity }\end{array}$ & $\begin{array}{l}\text { Encoding computing } \\
\text { power }\end{array}$ \\
\hline MPEG-1 & $8 \mathrm{Mbps}$ & Low & Small (x386) \\
\hline MPEG-2 & $5 \mathrm{Mbps}$ & Medium & Medium (x486) \\
\hline MPEG-4 & $\begin{array}{l}\text { 3-6 Mbps } \\
\text { (variable) }\end{array}$ & Medium & High (x586) \\
\hline H.264 & 1 Mbps & High & Very high (Dual core) \\
\hline
\end{tabular}

The reduction of transmitted information is usually based on unification of similar colours or not showing the details in similar colours. The most popular way of coding is the MPEG standard family, created by ISO organization (International Standards Organisation). Table 2 shows the basic characteristics of compression for particular versions of MPEG encoding and PAL television signal. All of MPEG standards are 
characterized by asymmetric computational requirements - the decoder part is much less complicated then the encoder, and requires only a small fraction of its computational requirements. This characteristic is one of main reasons of MPEG standards family practical popularity.

Table 3 shows a comparison of network bandwidth required in case of MPEG2/H.262 (currently most popular solution) and MPEG-4/H.263 (which is rapidly gaining popularity) employed in case of high definition video: 4CIF resolution (704 $\mathrm{x}$ 576) - the highest employed in industrial monitoring. In our research we decided to concentrate on variable rate stream and high quality, as a most resource intensive and difficult to effectively transmit type of video traffic.

The MPEG-4/H.263 transmission rate is variable in time, as it depends on changes in motion. MPEG-2/H.262 bandwidth is mostly constant and it is currently the most popular compression method used for the digital television transmission and many new services, such as VoD (Video on Demand).

Another solution, namely H.264 is the most sophisticated way of video encoding available currently and it is gaining a great popularity in television transmission and video conferences through cell phones. Its downside is a very high computational power necessary for encoding and high sensitivity to transmission errors, which makes it poorly suited for employment in a wireless environment.

Apart from the smallest possible output bandwidth, a good codec intended for streaming transmission should also provide a decent resistance to stream errors, which can result from malformed, lost or reordered IP datagrams. It is especially important in case of wireless transmissions, where probability of such errors is much higher than in case of cable-based transport technologies.

Based on literature study and some preliminary experiments, we decided to choose MPEG-4/H.263 codec as the subject of our detailed research. It offers a good compression ratio, acceptable resistance to streaming errors and can be employed in case of variety of video signals - starting from low quality mobile-phone video, and ending with high resolution, full motion TV.

Table 3. Comparison of bandwidth (in kbps) required by MPEG-2 and MPEG-4 encoding in closed-circuit television for 4CIF resolution, 25 frames per second, without sound, based on [3]

\begin{tabular}{|l|c|c|c|c|}
\hline \multirow{2}{*}{$\begin{array}{c}\text { 4CIF (704x576 pixels) } \\
25 \text { frames per second }\end{array}$} & MPEG2/ & \multicolumn{3}{|c|}{ X-series MPEG4/H.263 } \\
\cline { 3 - 5 } & Auto & $\begin{array}{c}\text { Highest } \\
\text { compression }\end{array}$ & $\begin{array}{c}\text { Medium } \\
\text { compression }\end{array}$ & $\begin{array}{c}\text { Highest } \\
\text { quality }\end{array}$ \\
\hline Many changes in image & 5000 & 1700 & 2950 & 4200 \\
\hline Partially changing image & 3500 & 950 & 1725 & 2500 \\
\hline No changes in image & 2000 & 700 & 1550 & 2400 \\
\hline
\end{tabular}

Another important element of real-time streaming system is data buffering. It allows the proper (constant and regular) timing of succeeding video frames display along with correct synchronization of sound. Experiences from practical usage of video coding and decoding applications show that the size of buffer is based on bandwidth of the network link and parameters of a video stream, such as its bandwidth and overall amount of data to be transmitted. 


\section{Network Transmission: Unicast (1:1) and Multicast (1:n)}

The most popular transmission protocol utilized for video streaming in IP network is RTP (Real Time Protocol). It is an unreliable transport protocol based on well-known UDP (User Data Protocol), extended with a number of mechanisms designed specifically for real-time, inelastic data transmission.

RTP can be used in both unicast and multicast streaming - each of these approaches offers unique advantages, but also brings specific requirements.

The unicast traffic stream delivers information to one particular receiver (point to point). Every new unicast connection causes the increase of overall bandwidth usage, which is proportional to the number of unicast streams present. An advantage of unicast transmission is that it can be initiated on demand of the user - that means, that:

- the encoder can be idle if there are no requests,

- each user can negotiate different stream characteristics,

- each user can control the content of its own stream.

In contrast, a single multicast stream delivers information to the group of receivers at once. The encoder is operating constantly and interested users can joint multicast session to receive the content that is currently being transmitted. Multicast is an effective way of sending the data from a single source to many receivers in a network [20]. In order to set up multicast transfer it is necessary to fulfil the following criteria:

- the video transmitter must be able to send the multicast streams,

- the receiver should be able to receive the multicast transmission,

- in order to receive multicast transmission the recipient has to join the particular multicast group - Internet Group Management Protocol (IGMP) signalisation support is required [20],

- to properly route multicast traffic in complex network environment, dedicated multicast routing protocol (for example: DVMRP, MOSPF, PIM-DM, PIM-SM...) must be implemented in routers [20].

\section{Test-Bed Environment}

Today, many popular devices, such as notebooks, laptops, palmtops and smart-phones implement an IEEE 802.11 compliant interfaces.

Most often, popular wireless LAN installations employ IEEE 802.11b/g compliant hardware, and there is also a growing number of IEEE 802.11n Draft 2 compliant devices.

Devices operating according to the IEEE 802.11a standard are not very popular among Small Office Home Office (SOHO) users, but they occupy an important place as short range infrastructure links in more complex wireless networks. Because of similarities between $802.11 \mathrm{a}$ being and $802.11 \mathrm{~g}$ standards, the empiric research was performed for the $802.11 \mathrm{~g}$ standard instead and the obtained results should be valid in both cases. 
Table 4. Features of particular $802.11 \mathrm{~b} / \mathrm{g} / \mathrm{n}$ standards

\begin{tabular}{|c|c|c|c|}
\hline Parameters & $\begin{array}{c}\text { Standard } \\
\mathbf{8 0 2 . 1 1 b}\end{array}$ & $\begin{array}{c}\text { Standard } \\
\mathbf{8 0 2 . 1 1 g}\end{array}$ & $\begin{array}{c}\text { Draft of standard } \\
\mathbf{8 0 2 . 1 1 n}\end{array}$ \\
\hline Year of issue & 1999 & 2003 & Draft - version no. 3 \\
\hline $\begin{array}{c}\text { Frequency } \\
\text { range }[\mathrm{GHz}]\end{array}$ & $2,4000 \div 2,4835$ & $2,400,0 \div 2,4835$ & $\begin{array}{c}2,4 \div 2.485 ; \\
5,470 \div 5,850 ;\end{array}$ \\
\hline $\begin{array}{c}\text { Channel bit } \\
\text { rate [Mbps] }\end{array}$ & 11 & 54 & $100 \div 300($ MCS15) \\
\hline $\begin{array}{c}\text { Modulation } \\
\text { technique }\end{array}$ & DQPSK. DBPSK & $\begin{array}{c}\text { BPSK, QPSK, } \\
\text { 16-QAM, 64-QAM }\end{array}$ & $\begin{array}{c}\text { Packet aggregation: A- } \\
\text { MPDU, A-MSDU }\end{array}$ \\
\hline $\begin{array}{c}\text { Transmission } \\
\text { technique }\end{array}$ & DSSS, CCK & OFDM & $\begin{array}{c}\text { OFDM+MIMO } \\
2 \times 2,2 \times 3,3 \times 3 \text { antennas }\end{array}$ \\
\hline
\end{tabular}

\section{Hardware Configuration}

Figure 1 presents a hardware configuration of our testbed. It consists of wired infrastructure and a stationary, IEEE 802.11 compliant, wireless access network working in an infrastructure mode.
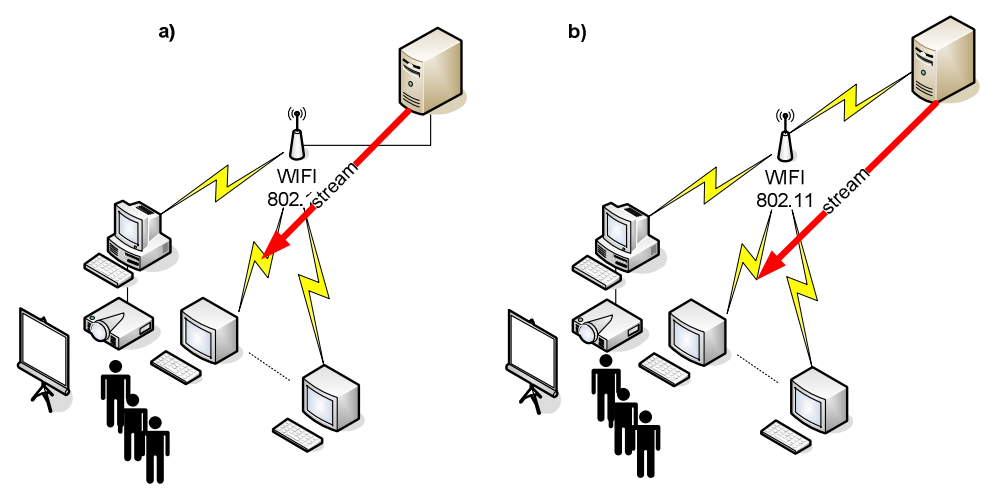

Fig. 1. WiFi network topology proposed with Access Point for empiric research. a) Cable to $\mathrm{WiFi}$, b) WiFi to WiFi.

The measurements were conducted two configurations:

- The video streaming source was located in the cable network. Video stream was sent through a simple cable infrastructure (consisting of a single Fast-Ethernet point-to-point link) to an Wireless Access Point and then to wireless IEEE 802.11 compliant clients.

- The video streaming source is connected as a wireless network client. The stream of video data is transmitted to clients exclusively by IEEE 802.11 network operating in an infrastructure mode. 
Different models of access points were tested. In all cases standard antennas built in the access point had been used:

- 802.11b - Linksys BEFW11S4v4,

- 802.11g - Linksys WAP54G-v3,

- $802.11 \mathrm{n}$ - Dlink DIR-655.

All clients were located in a room with size of about 25 square meters, lacking significant signal propagation barriers. Such environment provided good propagation conditions, characteristic to a well designed WLAN network in internal office spaces.

The radio channel has been verified as unused and lacking significant noise level during experiments. The transmission was conducted without encryption. Based on literature study and our earlier experiments, we can state that encrypting has a minimal influence on speed decrease in the transmission efficiency (about 5\%) and as such it doesn't have a significant influence on a video transmission [18].

\section{Software Configuration}

Video LAN Connector application [22] in version 0.8.6f has been used as both streaming server and client. WireShark Network Protocol Analyzer [21] has been employed as an measurement and analysis tool.

Both servers and client computers were working under control of Microsoft Windows XP SP2 operating system. Client computers were equipped with DualCore, Athlon, Celeron processors. Some small differences were observed in video streaming-related performance of particular computers, but they did not have a significant influence on the perception quality.

\section{Quality of Preception/Excellence Evaluation (QoP/E)}

Growing interest in multimedia applications, like Voice over IP (VoIP), Video on Demand (VoD) or interactive games, stimulates development of methods and tools designed for assessment of audio and video stream reception quality. They take into account both objective and subjective metrics - parameters leading to estimation of the level of recipients satisfaction, so-called Quality of Excellence (QoE).

Objective methods for audio signals include, among others: PEAQ [10], PSQM [12] and PESQ [16] algorithms, proposed by ITU that take into account from 5 up to 11 measured parameters.

For video signals Peak Signal to Noise Ratio (PSNR), ANSI [1] and J144 [15] methods are recommended.

At the same time a variety of subjective methods are proposed. However, the most popular are still relatively simple methods. For both audio and video signals Absolute Category Rating (ACR) [14] or Degradation Category Rating (DCR) [13] are often employed. Both methods use 5-degree grading tables.

In our research we decided to employ a subjective assessment method, supplemented by statistical traffic analysis conducted with mechanisms available in Wireshark Network Analyzer (such as stream bandwidth, packet delta, jitter and packet loss rate). 
A group of 10 test subjects has been selected. The scale of marks ranged from no degradation (mark 5) to very severe degradation (mark 1). The quality of received image has been measured according to values described in Table 5. Based on marks given by testers' so-called MOS (Mean Opinion Score) final parameter values were calculated. The average mark equal to 5 means excellent quality, while 1 - not acceptable. The observations of video quality were performed on client computers by users with no previous knowledge of the original material (so-called QoP method Quality of Perception estimation). The one-stimulus (eyesight) method has been used in which a group of respondents estimated the succeeding videos [7].

Table 5. Subjective qualitative estimation of video stream

\begin{tabular}{|r|l|l|}
\hline QoP & Levels of quality & Subjective impressions \\
\hline $\mathbf{5}$ & Excellent & $\begin{array}{l}\text { Imperceptible differences between local transmission and } \\
\text { the one after transmission remote access to a video stream }\end{array}$ \\
\hline $\mathbf{4}$ & Above Average & $\begin{array}{l}\text { Perceptible delay of transmission, but not affecting the } \\
\text { final reception }\end{array}$ \\
\hline $\mathbf{3}$ & Average & Small errors, temporary image freezing up to 1 second \\
$\mathbf{2}$ & Below average & Plenty of errors, image freezes for more than 1 second \\
\hline $\mathbf{1}$ & Fail & No image, or unrecognisable \\
\hline
\end{tabular}

The experiment result form (completed by test subjects) included the name of configuration scenario and video sample, time of measurement and the estimation. The estimations from all experiments of particular scenario were summarized thus receiving the average estimation of measurement. During the research, measurements of the transmission speed, jitter, packet lost and packet errors had also been conducted. The experiments were performed many times for the same scenario, in different rooms. The video material used during measurements was a PAL video recording encoded in the MPEG-4/H.263 standard where bandwidth of the stream was changing between 3 and $6 \mathrm{Mbps}$.

\section{Results}

In course of experiments conducted in our testbed installation, we were aiming to answer to the following basic questions:

1. How well various $\mathrm{WiFi}$ devices are prepared for transporting multicast and unicast real-time video streams?

2. What subjective quality of the video we can expect from a stream transported through the different WiFi network configurations and devices?

To assess the number of high definition (5 Mbps) MPEG4/H.263 video streams that a given 802.11 technology can support, we started with a simple network throughput assessment. 
A $10 \mathrm{MB}$ file has been transferred from the cable network to wireless client by unicast traffic, and transmission time, overall throughput and its stability has been measured - results are presented in Figure 2.

As we can see $802.11 \mathrm{~b}$ does not provide a sustained throughput required for even one of such high-definition video streams. 802.11g provided stable bandwidth of about 23 Mbps which can be enough for roughly 4-5 streams. 802.11n effective bandwidth was much less stable than in case of previous technologies, but, with mean rate of over $60 \mathrm{Mbps}$, it has a potential ability to support a significant number of hidef video streams.

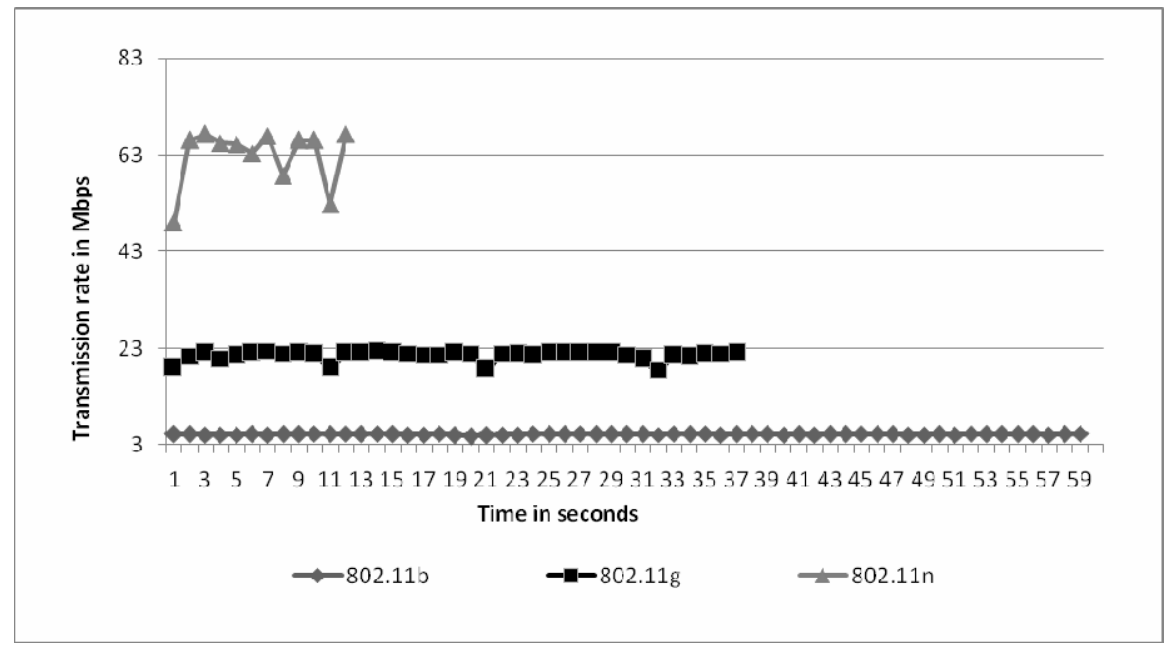

Fig. 2. Transfer time and transmission rate for $10 \mathrm{MB}$ file sent from cable network to a WiFi wireless client $(802.11 \mathrm{~b} / \mathrm{g} / \mathrm{n}$ standards)

Following this preliminary assessment, the main group of experiments has been conducted according to QoP evaluation rules described above. All popular WiFi base technologies were tested: IEEE 802.11 b, IEEE 802.11g (which earlier research describes as equivalent to IEEE 802.11a in our environment) and IEEE 802.11n Draft 2. Each technology has been tested for cable-wireless and wireless-wireless streaming. Both unicast and multicast tests were conducted.

The results of QoP evaluation are presented in Table 6.

The results of our experiments allow us to formulate the following direct conclusions:

1. IEEE $802.11 \mathrm{~b}$ standard cannot support hi-def video streaming.

2. IEEE $802.11 \mathrm{~g}$ based network supports up to 5 hi-def unicast video streams of acceptable quality.

3. IEEE $802.11 \mathrm{n}$ standard can support up to 5 independent hi-def video multicast streams.

One of the most significant results is a very profound difference in QoP scores between unicast and multicast streams for $802.11 \mathrm{~g}$ standard. 
Table 6. Summary of QoP evaluation

\begin{tabular}{|c|c|c|c|c|c|c|c|}
\hline \multirow{2}{*}{\multicolumn{2}{|c|}{$\begin{array}{l}\text { Number of concurrent } \\
\text { MPEG4-compressed } \\
\text { streams }\end{array}$}} & \multicolumn{6}{|c|}{$\begin{array}{c}\text { The WiFi standard and configuration of settings in } \\
\text { network of transmitters and receivers }\end{array}$} \\
\hline & & \multirow{2}{*}{$\begin{array}{c}802.11 b \\
\text { WiFi to } \\
\text { WiFi } \\
1,0 \\
\end{array}$} & \multirow{2}{*}{$\begin{array}{c}802.11 b \\
\text { Cable to } \\
\text { WiFi } \\
1,0\end{array}$} & \multirow{2}{*}{$\begin{array}{c}802.11 g \\
\text { WiFi to } \\
\text { WiFi } \\
1,5 \\
\end{array}$} & \multirow{2}{*}{$\begin{array}{c}\begin{array}{c}802.11 g \\
\text { Cable to } \\
\text { WiFi }\end{array} \\
1,0 \\
\end{array}$} & \multirow{2}{*}{$\begin{array}{c}802.11 n \\
\text { WiFi to } \\
\text { WiFi } \\
5,0 \\
\end{array}$} & \multirow{2}{*}{$\begin{array}{c}\begin{array}{c}802.11 n \\
\text { Cable to } \\
\text { WiFi }\end{array} \\
5,0 \\
\end{array}$} \\
\hline \multirow{9}{*}{ 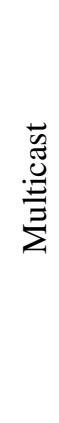 } & 1 stream & & & & & & \\
\hline & 2 streams & & & & & 4,7 & 5,0 \\
\hline & 3 streams & & & & & 4,3 & 5,0 \\
\hline & 4 streams & & & & & 3,9 & 5,0 \\
\hline & 5 streams & & & & & 3,8 & 4,7 \\
\hline & 6 streams & & & & & 2,9 & 4,4 \\
\hline & 7 streams & & & & & & 4,3 \\
\hline & 8 streams & & & & & & 4,1 \\
\hline & 9 streams & & & & & & 3,1 \\
\hline \multicolumn{2}{|c|}{$\begin{array}{l}\text { Average real speed in } \\
\text { Mbps }\end{array}$} & 2,76 & 5,41 & 13,34 & 19,45 & 20,52 & 62,9 \\
\hline \multirow{10}{*}{ 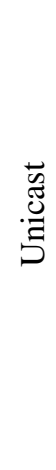 } & 1 stream & 1,8 & 4,2 & 5,0 & 5,0 & 5,0 & 5,0 \\
\hline & 2 streams & 1,0 & 3,6 & 5,0 & 5,0 & 5,0 & 5,0 \\
\hline & 3 streams & & 1,7 & 5,0 & 5,0 & 5,0 & 5,0 \\
\hline & 4 streams & & & 5,0 & 5,0 & 5,0 & 5,0 \\
\hline & 5 streams & & & 4,0 & 4,9 & 5,0 & 5,0 \\
\hline & 6 streams & & & 2,3 & 4,7 & 4,9 & 5,0 \\
\hline & 7 streams & & & 1,3 & 4,7 & 4,7 & 5,0 \\
\hline & 8 streams & & & & 4,5 & 4,4 & 5,0 \\
\hline & 9 streams & & & & 4,3 & 4,3 & 5,0 \\
\hline & 10 streams & & & & 3,9 & 3,8 & 5,0 \\
\hline \multicolumn{2}{|c|}{$\begin{array}{l}\text { Number of parallel } \\
\text { video transmissions in } \\
\text { PAL quality }\end{array}$} & 0 & 1 & 3 & 4 & 5 & 12 \\
\hline
\end{tabular}

By employing equipment from the year 2006, 5 parallel, unicast video steams were possible between wired LAN and WiFi.

This result is easy to predict theoretically, because by using the unicast transmission, we increase network load with each sender-recipient pair: 5 parallel transmissions, approximately $5 \mathrm{Mbps}$ each, take about $25 \mathrm{Mbps}$ of bandwidth, which is consistent with maximum bandwidth available for streaming with this technology. Above this limit, with each unicast stream created, the image quality was depreciating for all concurrent transmissions.

In case of multicast streams the situation is not obvious - it is notable, that even a single multicast stream transmitted through otherwise not utilized wireless network is of very bad quality. 
Broadcast and multicast frames, which can also be referred to as group frames (because they are destined for more than one receiving station) are exchanged without confirming acknowledgements. The sender has no way of confirming the success of transmission and will not retransmit group addressed frame. I other words, broadcast and multicast frames are delivered without any reliability guarantees or even indications of failure.

Moreover, while unicast traffic is transmitted with transmission rates dependant on current radio conditions and ranging from $1 \mathrm{Mbps}$ through $11 \mathrm{Mbps}$ (802.11b) to 54 Mbps (802.11 a/g, and even faster for 802.11n), group addressed frames are transmitted with rates from a much smaller set.

Each BSS maintains a list of transmission rates, which are supported by both ALL devices in a given BSS - a Basic Rate Set (BRS). Control and mulitcast/broadcast data frames may be transmitted only with rates that are listed in BRS, as they must be received and understood by all (or at least by a significant group) of the stations in the BSS [17]. The BRS is broadcasted by an AP controlling a given BSS and only stations supporting all its rates are allowed to join the network.

The default list of basic rates depends on implementation, but they rarely exceed 11 Mbps.

Theoretically a station or AP can choose any of BRS rate to transmit a group addressed frame, but often devices choose the slowest one, to maximize chances of successful transmission in absence of acknowledgement mechanism.

For example an unmodified Linksys WAP54G chooses $1 \mathrm{Mbps}$ rate. It is far too slow for streaming high-definition video. Moreover, we cannot change BRS set or rules for selecting transmission rates in a vast majority (probably about 90\%) of home use devices.

Table 7 presents statistical information obtained with the Wireshark software at receiver station. The first stream was transmitted with multicast frames at the default $1 \mathrm{Mbps}$. That speed is used in most of home use 802.11g APs for delivery of group addressed frames. In such transmission we lost $72 \%$ of transmitted multicast frames. Next we tested high level, professional AP from 3Com, model 8760 where we can select speed of multicast.

From our experiments, the highest rate we could choose in our environments, before transmission errors will offset throughput-based PoE advantages is $11 \mathrm{Mbps}$. We have only $2,1 \%$ of packet loss and mean jitter $2,1 \mathrm{~ms}$. [19]. That is a significantly

Table 7. Statistics of a single stream transmission in IEEE 802.11g standard. (3COM model $8760 \mathrm{AP})$.

\begin{tabular}{|c|c|c|c|c|c|c|}
\hline $\begin{array}{c}\text { Speed } \\
\text { Transmission } \\
\text { Type } \\
\text { (for 802.11g) }\end{array}$ & $\begin{array}{c}\text { Received } \\
\text { Packets } \\
\text { Number }\end{array}$ & $\begin{array}{c}\text { Lost } \\
\text { Packets } \\
(\text { percent })\end{array}$ & $\begin{array}{c}\text { Max } \\
\text { Delta } \\
(m s)\end{array}$ & $\begin{array}{c}\text { Max } \\
\text { Jitter } \\
(m s)\end{array}$ & $\begin{array}{c}\text { Mean } \\
\text { Jitter } \\
(m s)\end{array}$ & $\begin{array}{c}\text { QoP } \\
\text { score }\end{array}$ \\
\hline $\begin{array}{c}\text { 1Mbps } \\
\text { Multicast }\end{array}$ & 4694 & $\begin{array}{c}12033 \\
(71.9 \%)\end{array}$ & 94.90 & 88.74 & 5.33 & 1 \\
\hline $\begin{array}{c}\text { 11Mbps } \\
\text { Multicast }\end{array}$ & 16336 & $\begin{array}{c}347 \\
(2.1 \%)\end{array}$ & 200.27 & 41.78 & 2.91 & 3 \\
\hline $\begin{array}{c}\text { 54Mbps } \\
\text { Unicast }\end{array}$ & 16655 & $7(0.0 \%)$ & 194.94 & 24.18 & 4.23 & 5 \\
\hline
\end{tabular}


better result and allows us to successfully conduct video streaming. In the comparison case of $802.11 \mathrm{~g}$ unicast streams we have a more significant mean jitter, but just only 7 lost packets.

The new IEEE 802.11 standard extension - IEEE 802.11n in its current form of Draft 2 implementation, was first tested in an ad-hoc configuration (802.11n D-link external WiFi network adapters) and the maximum measured unicast transmission rate did not exceed $1 \mathrm{Mbps}$. The most probable reason for such a small bandwidth can be attributed to a very early firmware version present in that hardware. We hope that in next generation of firmware for $802.11 \mathrm{n}$ ad-hoc this will be corrected.

For the IEEE 802.11n network in infrastructure mode, the unicast transmission safely supported 9 parallel video streams between wireless stations. The overall consumed bandwidth reached $50 \mathrm{Mbps}$ and still all transmissions were of a very good quality. The further increase of streams was stopped, due to the limited number of available receiver-sender hardware device pairs.

Overall results (see figure 3) show, that regardless of employed technology we can always transmit more independent unicast streams than similar multicast streams. On the other hand, number of simultaneous unicast receivers is limited to the number of unicast streams, while number of multicast receivers is limited only by overall AP client capacity. IEEE 802.11g (Modified) corresponds to a IEEE 802.11g technology, where BRS has been modified (extended) to better accommodate multicast video streaming.

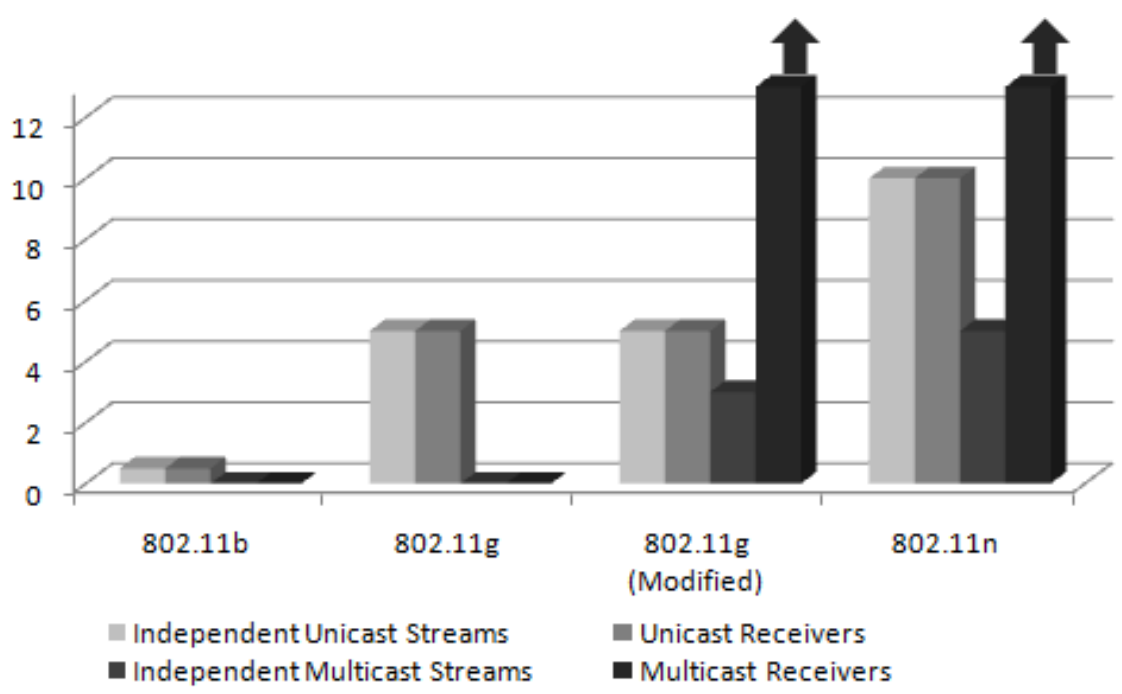

Fig. 3. Number of possible independent hi-def video streams and maximum number of receivers in case of various IEEE 802.11 technologies

\section{Conclusions}

The research proved that the real, effective rate of transmission of a given stream has a great influence on the quality of video material received from a WiFi network. The 
available network bandwidth should be enough to fully accommodate the needs of a video stream. Even slight deficiencies here result in drastic degradation of user experience (perception).

In case of unicast transmission this requirement is a straightforward one, despite the fact, that each client requires a separate point-to-point stream, so the number of simultaneous clients will be limited. Unicast WiFi traffic can easily utilize any transmission rate negotiated between AP and a client station, up to the maximum transmission rate. It makes it easy to assess the number of supported streams, and can be function on any popular WiFi hardware.

Multicast streaming has the undeniable advantage that the same material can be simultaneously provided to (theoretically) unlimited number of users which are in the range of AP's radio transmission. Unfortunately correct operation of this functionality depends on the particulars of AP implementation, and should not be considered advisable until it is verified for a particular model. That property results in serious differences in results obtained with use of simulation models and real life performance of particular devices. Implementation details of a particular device can cause differences in 0.3-2.5 times range (our estimation based on experimental research), between simulated performance and performance obtained in production environment.

IEEE 802.11 standard limits multicast transmission rates to a very limited rate set (rates < $11 \mathrm{Mbps}$ ), and practical implementations trend to choose the most stable (slowest) rate from this set resulting in $1 \mathrm{Mbps}$ multicast streaming bandwidth. For example device Linksys model WAP54G (manufactured in year 2006) is using the slowest of possible basic speed for $802.11 \mathrm{~g}$ multicast (1 Mbps). The new Access Point model 8760 from 3COM (manufactured in 8/8/2008) sets this speed, depending on radio conditions, on up to $11 \mathrm{Mbps}$. This is enough for just one stream encoded in MPEG4/H.263.

Some AP implementations offer possibility of changing rate set to be used for multicast and broadcast transmission, and that can provide us with considerable multicast bandwidth - such APs are highly advisable for multicast video streaming. However, we need to keep in mind, that WiFi group addressed frames are not acknowledged and we should keep multicast rate low enough to prevent an excessive loss of frames.

The IEEE 802.11n standard is a future of WiFi networks and, even in its current draft version, provides high bandwidth and ability to effectively handle both unicast and multicast MPEG-4 streams. There are still many upcoming changes, and today's implementations are likely to contain various errors (ad-hoc mode support, for example), but new transmission mechanisms present in this standard provide large advantages. Bandwidth and reliability of 802.11n, combined with 802.11e QoS mechanisms recently incorporated into main 802.11 standard, are going to provide us with a completely new environment for real-time wireless streaming. They are also the mechanisms, which we consider for further detailed testing.

\section{References}

1. ANSI T1.801.01-1996: Digital transport for video teleconferencing/ video telephony signals - Video test scenes for subjective and objective performance assessment, American National Standards Institute (1996) 
2. Bejerano, Y., Dongwook, L., Sinha, P., Zhang, L.: Approximation Algorithms for Scheduling Real-Time Multicast Flows in Wireless LANs. In: The 27th Conference on Computer Communications (INFOCOM 2008), Ohio State University, April 2008, pp. 2092 2100 (2008)

3. Spread calculation used to estimate the bandwidth and space on disk used for planning infrastructure with the $\mathrm{X}$-series devices coders made by the Bosh Corporation, Calculation made in July 22 (2005)

4. Chen, A., Dongwook, L., Sinha, P.: Optimizing Multicast Performance in Large-Scale WLANs, Ohio State University (2007)

5. Dujovne, D., Turletti, T.: Multicast in 802.11 WLANs: An Experimental Study, Project Planete INRIA, France (October 2006)

6. Fibush, D.K.: A Guide to Digital Television Systems and Measurments. Tektronix (1997)

7. Ghinea, G., Thomas, J.P., Fish, R.S.: Quality of perception to quality of service mapping using adynamically reconfigurable communication system. This paper appears in Conf. on Globecom 1999, Brazil, pp. 2061-2065 (1999)

8. Huynh-Thua, Q., Ghanbari, M.: Impact of Jitter and jerkiness on perceived video quality., Research made in University in Essex. Presented on Fourth International Workshop on Video Processing and Quality Metrics for Consumer Electronics (VPQM 2009), p. 308 (2006)

9. Ikkurthy, P.C.: Software testing testbed for mpeg-4 video traffic over ieee $802.11 \mathrm{~b}$ wireless lans. University of South Florida (2003)

10. ITU-R Recommendation BS 1387:Method for Objective Measurements of Perceived Audio Quality (PEAQ) (1998)

11. ITU-R Recommendation BT.1208-1: Video coding for digital terrestrial television broadcasting (October 1997)

12. ITU-T P.861: Objective Quality Measurement of Telephone-Band Speech Codecs (February 1998)

13. ITU-T P.910: Subjective video quality assessment for multimedia applications (1996)

14. ITU-T Rec. P.800: Method for subjective determination of transmission quality (1996)

15. ITU-T Recommendation J.144: Objective perceptual video quality measurement techniques for digital cable television in the presence of a full reference (March 2004)

16. ITU-T Recommendation P.862: Perceptual evaluation of speech quality (PESQ), an objective method for end-to-end speech quality assessment of narrowband telephone networks and speech codecs (1998)

17. Gast, M.: 802.11 Wireless Networks The Definitive Guide. O’Reilly, Sebastopol (2005)

18. NetGear: Wireless Performance Study. LioNBridge (October 2006), http: / / www . veritest.com

19. Schulzrinne H., Casner S., Federick R., Jacobson V.: RTP: A Transport Protocol for RealTime Applications, RFC 3550 (June 2003)

20. Wittmann, R., Zitterbart, M.: Multicast Communication: Protocols and Applications. Morgan Kaufmann Publisher, translated by Academic Press (2001)

21. http://www. wireshark.org/

22. http://www. videolan.org/vlc/ 